\title{
O setor produtivo de flores e plantas ornamentais do Brasil, no período de 2008 a 2013: atualizações, balanços e perspectivas ${ }^{(1)}$
}

\author{
ANTONIO HÉLIO JUNQUEIRA (2) E MARCIA DA SILVA PEETZ (3)
}

\begin{abstract}
RESUMO
A floricultura empresarial brasileira constitui-se em um dos mais dinâmicos e promissores segmentos do agronegócio brasileiro contemporâneo, exibindo indicadores de crescimento significativos, tanto em termos de número de produtores, quanto de área cultivada e de Valor Bruto da Produção (VBP). O presente artigo revisa e atualiza dados nacionais e macrorregionais destes indicadores, a partir de uma extensa e exaustiva pesquisa de dados estatísticos e análises geradas junto a empresas e órgãos públicos e privados ligados ao setor. Oferece, assim, um panorama comparativo entre os anos de 2008 e 2013, apontando para alguns fenômenos tendenciais da organização produtiva do setor no País.
\end{abstract}

Palavras-chave: flores e plantas ornamentais, produção, área, Valor Bruto da Produção, regionalização

\section{ABSTRACT \\ The productive sector of flowers and ornamental plants of Brazil, in the period from 2008 to 2013: updates, balance sheets and prospects}

The Brazilian floriculture business is one of the most dynamic and promising segments of contemporary agribusiness, displaying indicators of significant growth, in terms of number of producers, cultivated area and Gross Value of Production (GVP). This article reviews and updates the national and macro-regional data from these indicators, from an extensive and exhaustive research of statistical data and analyzes generated from companies and public and private agencies connected with the industry. Thus provides a comparative overview between the years 2008 and 2013, pointing to some trend phenomena of productive sector organization in the country.

Keywords: flowers and ornamental plants, production area, Gross Value of Production (GPV), regionalization

\section{INTRODUÇÃO}

A cadeia produtiva de flores e plantas ornamentais no Brasil movimentou, no ano de 2013, o valor global de R \$ 5,22 bilhões, acumulando crescimento de $8,3 \%$ sobre os resultados obtidos no ano de 2012. Para 2014, as estimativas preliminares apontam para um total de $\mathrm{R} \$ 5,64$ bilhões, basicamente resultante de um novo crescimento de $8,0 \%$ sobre o ano anterior.

Tais resultados mostram-se positivos e economicamente alvissareiros, especialmente frente ao quadro de crescimento econômico tendente à estagnação, que já se desenha no cenário nacional. Ainda que tenha desacelerado frente às conquistas comerciais anteriores, a economia do comércio florícola brasileiro mostra-se aquecida em decorrência dos seguintes fatores principais: a) melhora contínua dos indicadores sociais de ocupação, emprego, renda e consumo de amplas parcelas da população brasileira, vigente especialmente ao longo do período de 2006 a 2013; b) aumento da penetração do varejo de autosserviço na distribuição de flores e plantas em todo o País, agregando ao setor a capilaridade da rede comercial, a hiperexposição das mercadorias, o incremento dos apelos e das vendas por impulso e a redução dos preços finais aos consumidores, dados os ganhos de escala e poder de barganha no mercado; c) expansão e aperfeiçoamento da rede de comércio atacadista, com a oferta de instalações ampliadas, aumento do uso da cadeia do frio e agregação de novos serviços aos fornecedores e clientes e d) expansão da oferta regional de mercadorias, em decorrência tanto do crescimento e do fortalecimento de polos produtivos regionais, quanto do redirecionamento, para o mercado interno, de produtos de exportação, face à conjuntura recessiva do mercado mundial, instaurada a partir do ano de 2008.

Neste contexto, um novo levantamento estatístico realizado pela empresa Hórtica Consultoria e Treinamento, para o ano de 2013, a partir da coleta, compilação, sistematização e análise crítica das informações geradas por cooperativas e associações de produtores, mercados e centrais atacadistas, secretarias estaduais de agricultura, pecuária e abastecimento, órgãos públicos e privados afins, universidades e institutos de pesquisa de todo o País, permitiu realizar as análises decorrentes da evolução de indicadores, para os anos de 2008 e 2013.

\footnotetext{
(1) Trabalho recebido para publicação em 03/08/2014 e aprovado em 13/10/2014

(2) Engenheiro agrônomo, Doutor em Ciências da Comunicação (ECA/USP), Mestre em Comunicação e Práticas de Consumo (ESPM), Especialista em Desenvolvimento Rural e Abastecimento Alimentar Urbano (FAO/PNUD/CEPAL/IPARDES), sócio proprietário da Hórtica Consultoria e Treinamento helio@hortica.com.br.

(3) Economista, Especialista em Economia Agrícola, Desenvolvimento Rural Comercialização e Abastecimento Alimentar Urbano, sócia proprietária da Hórtica Consultoria e Treinamento.marcia@hortica.com.br
} 


\section{INDICADORES DE DESENVOLVIMENTO DA ATIVIDADE}

Os dados coletados e analisados permitiram concluir que existem, no Brasil, 7.800 produtores de flores e plantas ornamentais, os quais, em seu conjunto, cultivaram, em 2013, uma área total de 13.468 hectares.
Em termos macrorregionais, o Sudeste seguiu concentrando a maior parcela do número total de produtores, acumulando $53,3 \%$ do total contabilizado. Na sequência da ordem relativa de grandeza decrescente vieram as regiões Sul, com $28,6 \%$ de participação porcentual, Nordeste, com $11,8 \%$, Norte, ficando com $3,5 \%$ e, finalmente, Centro-Oeste, com representatividade de $2,8 \%$.

Tabela 1. Número de produtores e área cultivada com flores e plantas ornamentais no Brasil, em hectares, segundo macrorregiões geográficas, em 2008 e 2013.

Table 1 Number of producers and acreage with flowers and ornamental plants in Brazil, in hectares, according to macrogeographical regions in 2008 and 2013.

\begin{tabular}{|c|c|c|c|c|c|c|c|c|}
\hline \multirow{3}{*}{$\begin{array}{l}\text { MACRORREGIÕES } \\
\text { Macro-regions }\end{array}$} & \multicolumn{4}{|c|}{$\begin{array}{c}\text { Produtores } \\
\text { Producers } \\
\end{array}$} & \multicolumn{4}{|c|}{$\begin{array}{l}\text { Área Cultivada } \\
\text { Cultivated area } \\
\end{array}$} \\
\hline & \multicolumn{2}{|c|}{2008} & \multicolumn{2}{|c|}{2013} & \multicolumn{2}{|c|}{2008} & \multicolumn{2}{|c|}{2013} \\
\hline & $n^{0}$ & $\%$ & $n^{0}$ & $\%$ & ha & $\%$ & ha & $\%$ \\
\hline Sudeste & 3.611 & 60,0 & 4.158 & 53,3 & 7.651 & 64,2 & 8.869 & 65,9 \\
\hline \multicolumn{9}{|l|}{ Southeast } \\
\hline Sul & 1.220 & 20,3 & 2.229 & 28,6 & 2.763 & 23,2 & 2.914 & 21,6 \\
\hline \multicolumn{9}{|l|}{ South } \\
\hline Centro-Oeste & 157 & 2,6 & 220 & 2,8 & 302 & 2,5 & 372 & 2,8 \\
\hline \multicolumn{9}{|l|}{ Center-West } \\
\hline Nordeste & 808 & 13,4 & 923 & 11,8 & 959 & 8,0 & 1.023 & 7,6 \\
\hline \multicolumn{9}{|l|}{ Northeast } \\
\hline Norte & 227 & 3,8 & 270 & 3,5 & 241 & 2,0 & 290 & 2,2 \\
\hline \multicolumn{9}{|l|}{ North } \\
\hline BRASIL & 6.023 & 100,0 & 7.800 & 100,0 & 11.916 & 100,0 & 13.468 & 100,0 \\
\hline
\end{tabular}

Fonte: Hórtica Consultoria e Treinamento, 2014, a partir da coleta, consolidação, adaptação e projeções sobre estatísticas coletadas e divulgadas por: CEAGESP (2008-2013), CEASA CAMPINAS (2008-2013), CEASA PR (2008-2013), CEASA RS (2008-2013), COOPERATIVA VEILING HOLAMBRA (2008-2013), DAUDT (2002), EPAGRI-CEPA/SC (2008), ESPÍRIO SANTO (2006), IEA/CATI-SP (LUPA) (2007/2008), INSTITUTO AGROPOLOS DO CEARÁ (2010), FRANCISCO; PINO; KIYUNA (2003a e 2003 b), FUNKE; PEREIRA (2011), IBGE (2004), JUNQUEIRA; PEETZ (2002, 2005a, 2005b, 2005c, 2005d, 2006, 2007a, 2007b, 2008, 2010); LANDGRAF (2006), MATOS \& ALVES (2007), PADULA; KÄMPF; SLONGO (2003), RIO DE JANEIRO (2004, 2011, 2014a, 2014b), SEAB-DERAL/ PR (2008-2013), SEBRAE/AM (2008), SEBRAE/CE (2008), SEBRAE/PE (2002), SEBRAE/PI (2008); SEBRAE/RN (2008) e SEBRAE/RS (2003).

No tocante à área cultivada com flores e plantas ornamentais, o mesmo conjunto estatístico permitiu constatar a elevada concentração da atividade na região do Sudeste brasileiro, que ficou com $65,9 \%$ da superfície total cultivada com essas espécies. Para o mesmo ano de 2013, a região Sul agregou representatividade de $21,6 \%$ no total da área nacional, seguida pelo Nordeste, com $7,6 \%$ de participação na distribuição nacional da área florícola, seguido pelo Centro-Oeste, com $2,8 \%$ e pelo Norte, com $2,2 \%$.

A análise comparativa com série estatística anterior, disponível para o ano de 2008 (JUNQUEIRA; PEETZ, 2010), permite comprovar as seguintes características evolutivas e tendenciais da floricultura profissional brasileira:

1. Crescimento da participação de micro e pequenos produtores: no período de 2008 a 2013, o número de produtores dedicados ao cultivo de flores e plantas ornamentais no
Brasil elevou-se em 29,5\%, passando de 6.023, para 7.800. Entre esses mesmos anos considerados, o total da área cultivada exibiu crescimento significativamente mais modesto, de $13,0 \%$, saltando de um total de 11.916 hectares dedicados à atividade, em 2008, para 13.468, em 2013. Tal fenômeno resultou no fato de que a área média cultivada por produtor se reduzisse em 12,6\%, passando de 1,98 hectare por produtor, em 2008, para 1,73 hectare, em 2013;

2. Dinamismo diferencial entre macrorregiões geográficas: de uma maneira geral, entre os anos de 2008 e 2013, houve crescimento no número de produtores em todas as macrorregiões geográficas brasileiras. Porém, a intensidade desse crescimento foi significativamente diferenciada entre elas. Neste contexto, a região Sul foi a que acrescentou maior parcela expansiva, elevando o número de produtores em $82,7 \%$, que passou de 1.220, em 2008, para 2.229, em 2013. Na se- 
gunda posição, ficou o Centro-Oeste, com aumento de 40,1\% neste indicador, o qual elevou-se de 157 para 220 entre os anos acompanhados. No Norte, a expansão observada foi de 18,9\%; no Sudeste, de $15,1 \%$ e no Nordeste, de $14,2 \%$.

No caso da macrorregião geográfica Sul, o aumento substancial do número de produtores contabilizados deveu-se particularmente à situação observada para o Rio Grande do Sul que, entre os anos acompanhados, reviu suas estatísticas oficiais de 560 produtores, em 2008, para 1.519, em 2013, conforme os dados revelados pela Câmara Setorial da Cadeia Produtiva de Flores e Plantas Ornamentais do Estado do Rio Grande do Sul, por ocasião de sua instalação junto à Secretaria de Agricultura, Pecuária de Abastecimento daquele Estado, ocorrida no mês de maio de $2013^{(4)}$.

Já para o Nordeste, onde se observou o menor índice de dinamismo da atividade entre os anos considerados, os principais motivos encontrados referem-se primeiramente à retração do mercado importador mundial, para onde se vinham dirigindo grande parte dos esforços produtivos e dos investimentos setoriais e, em segundo, como consequência da incapacidade de reconversão para o mercado interno de parcelas substanciais da produção de flores e folhagens tropicais, haja vista que os índices de consumo destas mercadorias ainda são considerados baixos e pouco atraentes nos principais mercados consumidores do Sudeste e do Sul do País. Tais fenômenos impactaram negativamente a economia florícola regional, fazendo com que muitos produtores de maior porte desistissem de suas atividades, sendo substituídos, numericamente, por micro e pequenos produtores focados no abastecimento local e regional, sobretudo nos Estados da Bahia e da Paraíba;

3. Aumento da concentração macrorregional da área cultivada: os dados coletados e analisados permitem observar que, entre os anos de 2008 e 2013, houve crescimento ainda mais significativo da concentração da atividade produtiva na floricultura brasileira. De fato, o Sudeste - principal polo florícola nacional - aumentou sua participação porcentual relativa de $64,3 \%$, em 2008, para 65,9\%, em 2013. Tal fenômeno implicou a incorporação de novos 1.218 hectares, equivalentes a $9,0 \%$ de toda a área brasileira dedicada à produção de flores e plantas ornamentais e 78,5\% do total da nova área agregada à floricultura, que foi, entre os anos de 2008 e 2013, de 1.552 hectares. Ao mesmo tempo, as regiões Sul e Nordeste tiveram participação relativa decrescente decadente na distribuição proporcional deste indicador no período, passando, respectivamente, de $23,2 \%$ e $8,0 \%$ do total nacional, para $21,6 \%$ e $7,6 \%$. Finalmente, observou-se que as macrorregiões geográficas Centro-Oeste e Norte elevaram suas participações porcentuais relativas de $2,5 \%$ e $2,0 \%$, respectivamente, para $2,8 \%$ e $2,2 \%$.

\section{VALOR BRUTO DA PRODUÇÃO (VBP) DA FLORICULTURA}

Em termos de Valor Bruto da Produção (VBP) - ou seja, dos valores efetivamente recebidos pelos produtores de flores e plantas ornamentais -, a floricultura brasileira movimentou, em 2013, R\$ 1,49 bilhão, com crescimento de 57,56\% sobre os resultados apurados para o ano de 2008 ( $\mathrm{R} \$ 945.649$ milhões). A região Sudeste, liderada pelo Estado de São Paulo, agregou $73,74 \%$ de participação, confirmando o elevado grau de concentração da atividade florícola nacional. Em 2008, a participação porcentual relativa desta região representava $70,91 \%$ do total do VBP da floricultura. Internamente, cabe destacar que o Estado de São Paulo, principal produtor nacional, acumulou decréscimo de participação no período analisado, a qual decaiu de 52,81\%, para 48,31\%. Os Estados de Minas Gerais e do Espírito Santo mantiveram praticamente os mesmos porcentuais, enquanto o Estado do Rio de Janeiro galgou posições fortemente significativas, passando de 3,62\% para 11,05\% (RIO DE JANEIRO, 2011, 2014a, 2014b).

Internamente, o agronegócio de flores e plantas ornamentais do Brasil é segmentado majoritariamente na produção de espécies de plantas ornamentais para paisagismo e jardinagem, o qual concentrou, em 2013, 41,55\% do total do VBP (R\$ 619.049.000,00) não incluindo, neste montante, o segmento específico das gramas esportivas e ornamentais, que pode ser considerado autônomo e organizado segundo parâmetros próprios e específicos de produção, distribuição e consumo.

Na segunda posição em ordem de grandeza, definida pela participação porcentual relativa decrescente de importância, fica o setor de flores e folhagens de corte, com 34,33\%, seguido pelo das flores e plantas envasadas $(24,12 \%)$.

A análise comparativa com a série de dados anterior (JUNQUEIRA; PEETZ, 2010), de 2008, permite constatar que tanto as flores e folhagens de corte quanto as envasadas exibiram crescimento em suas participações porcentuais relativas. De fato, as primeiras elevaram seu índice porcentual de $31,41 \%$, em 2008 , para $34,33 \%$, em 2013 , enquanto que as flores e plantas envasadas o fizeram da proporção de $20,0 \%$ para $24,12 \%$. Tais crescimentos se deram em detrimento do maior segmento - o das plantas ornamentais para paisagismo e jardinagem - que, no mesmo período, reduziu sua representatividade porcentual de $48,59 \%$ para $41,55 \%$.

Para as flores e folhagens de corte, o crescimento relativo encontrado justifica-se pelo crescimento global do mercado interno para essas mercadorias, assim como também pelo aumento do número de produtores e da área a elas dedicada tanto nos novos polos de produção regional, especialmente no Norte, Nordeste e Centro-Oeste, quanto pela recuperação e ampliação dos polos produtivos tradicionais do Rio de Janeiro, Minas Gerais, Paraná e Rio Grande do Sul.

A produção nacional de flores e folhagens de corte, avaliada pelo VBP, concentra-se especialmente na região Sudeste que, em 2013, agregou a participação porcentual relativa de $83,02 \%$. Em sequência, se posicionaram as regiões Nordeste $(9,92 \%)$, Sul $(2,90 \%)$, Norte $(2,62 \%)$ e Centro-Oeste $(1,54 \%)$.

Já para as flores e plantas envasadas, os resultados apontam para uma gradual mudança nas preferências de consumo, que se alinham com tendências mundiais, segundo as quais essas mercadorias tendem a ganhar importância relativa sobre as flores e folhagens de corte. Tais constatações justificam-se pela percepção dos consumidores da existência

\footnotetext{
(4) ASSOCIAÇÃO RIO-GRANDENSE DE FLORICULTURA (Aflori). Panorama da floricultura no Estado do Rio Grande do Sul. Dados apresentados durante a cerimônia de instalação da Câmara Setorial de Flores e Plantas Ornamentais do Rio Grande do Sul, junto à Secretaria Estadual de Agricultura, Pecuária e Agronegócio (Seapa), em 30 de abril de 2013.
} 
Tabela 2. Valor Bruto da Produção (VBP), total e por setores da floricultura no Brasil, segundo macrorregiões geográficas, em 2013.

Table 2 Gross Value of Production (GVP), total and floriculture sectors in Brazil, according to macro-geographical regions in 2013.

\begin{tabular}{|c|c|c|c|c|c|c|c|}
\hline \multirow{4}{*}{$\begin{array}{l}\text { MACRORREGIÕES } \\
\text { Macro-regions }\end{array}$} & \multicolumn{7}{|c|}{$\begin{array}{l}\text { Valor Bruto da Produção (VBP) } \\
\text { Gross Value of Production (GVP) }\end{array}$} \\
\hline & \multirow{3}{*}{$\begin{array}{c}\text { Total } \\
\text { (R\$ mil) }\end{array}$} & \multicolumn{6}{|c|}{ Setores } \\
\hline & & \multicolumn{2}{|c|}{$\begin{array}{c}\text { Flores e folhagens } \\
\text { de corte } \\
\text { Cut flowers and } \\
\text { foliages } \\
\end{array}$} & \multicolumn{2}{|c|}{$\begin{array}{c}\text { Flores e plantas } \\
\text { envasadas } \\
\text { Pot plants }\end{array}$} & \multicolumn{2}{|c|}{$\begin{array}{c}\text { Plantas para paisagismo e } \\
\text { jardinagem } \\
\text { Plants for landscaping and } \\
\text { gardening } \\
\end{array}$} \\
\hline & & (RS mil) & $\%$ & (RS mil) & $\%$ & (RS mil) & $\%$ \\
\hline Sudeste & 1.098 .710 & 424.695 & 38,65 & 300.020 & 27,31 & 373.995 & 34,04 \\
\hline \multicolumn{8}{|l|}{ Southeast } \\
\hline Sul & 146.602 & 14.865 & 10,14 & 29.194 & 19,91 & 102.543 & 69,95 \\
\hline \multicolumn{8}{|l|}{ South } \\
\hline Centro-Oeste & 73.571 & 7.871 & 10,70 & 4.080 & 5,55 & 61.620 & 83,76 \\
\hline \multicolumn{8}{|l|}{ Center-West } \\
\hline Nordeste & 134.290 & 50.737 & 37,78 & 24.685 & 18,38 & 58.868 & 43,84 \\
\hline \multicolumn{8}{|l|}{ Northeast } \\
\hline Norte & 36.827 & 13.405 & 36,40 & 1.399 & 3,80 & 22.023 & 59,80 \\
\hline \multicolumn{8}{|l|}{ North } \\
\hline BRASIL & 1.490 .000 & 511.572 & 34,33 & 359.379 & 24,12 & 619.049 & 41,55 \\
\hline
\end{tabular}

Fonte: Hórtica Consultoria e Treinamento, 2014, a partir da coleta, consolidação, adaptação e projeções sobre estatísticas coletadas e divulgadas por: CEAGESP (2008-2013), CEASA CAMPINAS (2008-2013), CEASA PR (2008-2013), CEASA RS (2008-2013), COOPERATIVA VEILING HOLAMBRA (2008-2013), DAUDT (2002), EPAGRI-CEPA/SC (2008), ESPÍRIO SANTO (2006), IEA/CATI-SP (LUPA) (2007/2008), INSTITUTO AGROPOLOS DO CEARÁ (2010), FRANCISCO; PINO; KIYUNA (2003a e 2003 b), FUNKE; PEREIRA (2011), IBGE (2004), JUNQUEIRA; PEETZ (2002, 2005a, 2005b, 2005c, 2005d, 2006, 2007a, 2007b, 2008, 2010); LANDGRAF (2006), MATOS \& ALVES (2007), PADULA; KÄMPF; SLONGO (2003), RIO DE JANEIRO (2004, 2011, 2014a, 2014b), SEAB-DERAL/ PR (2008-2013), SEBRAE/AM (2008), SEBRAE/CE (2008), SEBRAE/PE (2002), SEBRAE/PI (2008); SEBRAE/RN (2008) e SEBRAE/RS (2003).

de uma melhor relação custo versus benefício para as flores e plantas envasadas, dados seus menores custos relativos, maior durabilidade e maior praticidade no uso decorativo e no manuseio doméstico ou no ambiente profissional. Frente à conjuntura econômica recessiva em amplitude mundial, as flores e plantas envasadas mostram maior potencial de penetração nas cestas de consumo e, face às condições da vida urbana contemporânea, adequam-se melhor à falta de tempo, deslocamentos e viagens constantes, habitações de tamanho reduzido e menor presença de acessórios domésticos, como vasos e utensílios necessários para o trato e arranjo das flores e folhagens cortadas.

$\mathrm{Na}$ floricultura nacional, as flores e plantas envasadas concentram seu cultivo especialmente na região Sudeste, com $83,48 \%$, sendo puxadas pelos ascendentes mercados de orquídeas, bromélias, kalanchoes, lírios, begônias, violetas e samambaias, entre outras espécies. A essa região seguem Sul $(8,12 \%)$, Nordeste $(6,87 \%)$, Centro-Oeste $(1,14 \%)$ e Norte $(0,39 \%)$.
Finalmente, no segmento das plantas ornamentais para paisagismo e jardinagem, basicamente puxado pelo dinamismo da indústria da construção civil, o maior índice de concentração macrorregional é também encontrado no Sudeste, que concentrou, em 2013, 60,41\% de participação porcentual relativa. Cabe observar, no entanto, que entre os três setores analisados, é o que mostra maior grau de dispersão geográfica, com maiores e mais significativas presenças das demais regiões, especialmente do Sul (16,57\%), capitaneado pelo estado do Paraná. Seguem a essa, Centro-Oeste $(9,95 \%)$, com destaque para o setor viveirista do Estado de Goiás, Nordeste $(9,51 \%)$ e Norte $(3,56 \%)$.

\section{CONSIDERAÇÕES FINAIS}

O setor produtivo de flores e plantas ornamentais no Brasil vem consistentemente consolidando posições mais relevantes no agronegócio nacional, destacando-se como atividade 
economicamente crescente, que além de agregar alto potencial de expansão futura, representa também uma das principais atividades geradoras de ocupação, emprego e renda para micro e pequenos produtores em todo o País, incorporando importantes parcelas do trabalho feminino rural.

Focada essencialmente no abastecimento do mercado interno, para o qual dirige $96,5 \%$ de todo o valor que produz, a floricultura brasileira tem seguido praticamente inabalável frente ao ambiente de crise econômica e financeira que atingem o mercado internacional desde 2008, sendo, desta forma, fator de estabilidade e confiança no campo.

Para os próximos anos, o cenário certamente continuará bastante favorável, ainda que se apontem tendências de redução na pujança do crescimento econômico. Neste contexto, caberá ao setor, de forma organizada e profissional, avançar na consolidação de suas conquistas, de modo que os futuros resultados sejam cada vez mais sustentáveis e permanentes.

Em relação a projeções futuras, com base nos indicadores atuais do mercado, figuram, sem dúvida, os seguintes pontos principais: a) fortalecimento e profissionalização crescentes das vendas de flores e plantas ornamentais pelo varejo de autosserviço (supermercados e garden centers); b) continuidade do crescimento e do fortalecimento do mercado de paisagismo e jardinagem, que continuará recebendo impulsos da indústria da construção civil; c) reorganização da base produtiva da floricultura nacional, com a continuidade do fortalecimento e estruturação crescente dos polos regionais; d) busca permanente de agregação de novos produtos, serviços e qualidade de mercadorias e atendimento, como forma de conquistar e manter clientes e consumidores, e d) revisões, redirecionamentos, novos rumos e ritmos no desenvolvimento das políticas setoriais de apoio e fomento, decorrentes dos rearranjos político-institucionais.

Como respostas principais a esses desafios, esperam-se dos empreendedores da floricultura brasileira: a) orientação da produção para as novas condições e exigências do mercado; b) agregação de valor aos produtos e serviços; c) profissionalização não apenas da produção, mas especialmente da pós-colheita, logística e distribuição; d) revisão e adequação permanente dos custos; e) fortalecimento das ações institucionais, associativistas e cooperativistas; f) intensificação das ações de promoção e marketing, e g) foco na atenção total à satisfação dos clientes, antecipando e superando seus desejos e necessidades.

\section{REFERÊNCIAS}

CAMPINAS (Município). Centrais de Abastecimento de Campinas S/A. Estatísticas de Comercialização do Mercado Permanente de Flores e Plantas Ornamentais, 2008 a 2014.

COMPANHIA DE ENTREPOSTOS E ARMAZÉNS GERAIS DE SÃO PAULO - CEAGESP: Séries estatísticas de comercialização no Mercado de Flores e Plantas Ornamentais, 2008-2014.
DAUDT, R.H.S. Censo da produção de flores e plantas ornamentais no Rio Grande do Sul/ Brasil na virada do milênio. 2002. 86 p. (Dissertação de Mestrado em Fitotecnia) - Faculdade de Agronomia da Universidade Federal do Rio Grande do Sul, Rio Grande do Sul, 2002.

ESPÍRITO SANTO (Governo do Estado). Secretaria de Estado da Agricultura, Abastecimento, Aqüicultura e Pesca. Plano Estratégico de Desenvolvimento da Floricultura do Estado do Espírito Santo (PEDEAG-FLORICULTURA), 2004. Disponível em: <http://www.seag.es.gov.br/floricultura.htm. $>$ Acesso em 28 de jul.2006.

FUNCKE, A. L.; PEREIRA, P. R. F. Perfil da cadeia produtiva de plantas ornamentais de Guratiba (RJ). SEBRAE RJ; Prefeitura Municipal do Rio de Janeiro, Brix Pace. Rio de Janeiro: SEBRAE RJ, abril de 2011 (Relatório de Pesquisa).

INSTITUTO AGROPOLOS DO CEARÁ. Diagnóstico da floricultura no Estado do Ceará 2009-2010. Fortaleza, CE: Instituto Agropolos, 2010.

FRANCISCO, V.L.F.S.; PINO, F. A.; KIYUNA, I. Floricultura no Estado de São Paulo. Informações Econômicas, São Paulo, v.33, n.3, p.17-32, 2003a.

FRANCISCO, V.L.F.S.; PINO, F.A.; KIYUNA, I. Os floricultores no Estado de São Paulo. Informações Econômicas, São Paulo, v.33, n.12, p.74-80, 2003 b.

FUNDAÇÃO INSTITUTO BRASILEIRO DE GEOGRAFIA E ESTATÍSTICA(IBGE). Caracterização do setor produtivo de flores e plantas ornamentais no Brasil, 1995-1996. Rio de Janeiro: IBGE, 2004. (Estudos e Pesquisas, informação econômica, n.2).

JUNQUEIRA, A.H.; PEETZ, M.S. Perfil da Cadeia Produtiva de Flores e Plantas Ornamentais do Distrito Federal. Brasília: SEBRAE / DF. (Edição SEBRAE), 121p., 2005a.

JUNQUEIRA, A.H.; PEETZ, M.S. Perfil da Cadeia Produtiva de Flores e Plantas Ornamentais da Mesorregião Metropolitana de Belém. Belém: SEBRAE/PA, 2006. Disponível em: <http://www.biblioteca.sebrae.com.br/bds/ BDS.nsf/4F8048F06CA79B1F03257222004FB603/\$File/ NT000B5D02.pdf.> Acesso em 01 jul.2006.

JUNQUEIRA, A.H.; PEETZ, M.S. Producción y comercialización de plantas ornamentales en Brasil. Horticultura Internacional, Tarragona, Espanha, ano XIV, n.55, p.16-19, 2007a.

JUNQUEIRA, A.H.; PEETZ, M.S. Acesso a Mercados: Perfil Mercadológico das Empresas. Projeto de Fortalecimento do Setor de Flores e Plantas Ornamentais do Estado do Espírito Santo. SEBRAE/ ES, $2007 b$. 
JUNQUEIRA, A.H.; PEETZ, M.S. Estudo da Competitividade e Eficiência da Cadeia Produtiva de Flores e Plantas Ornamentais na Região Norte do Brasil Amazônia. Manaus: SEBRAE AM., 2010.

JUNQUEIRA, A.H.; PEETZ, M.S. Os polos de produção de flores e plantas ornamentais do Brasil: uma análise do potencial exportador. Revista Brasileira de Horticultura Ornamental, Campinas, v.8, n. 1/2, p.25-48, 2002.

JUNQUEIRA, A.H.; PEETZ, M.S. Perfil da Cadeia Produtiva de Flores e Plantas Ornamentais no Estado do Pará. Belém: SEBRAE PA, 2005 b.

JUNQUEIRA, A.H.; PEETZ, M.S. Comercialização de flores tropicais brasileiras nos mercados interno e externo. Flores Tropicais. Fortaleza: Embrapa Agroindústria Tropical, $2005 \mathrm{c}$.

JUNQUEIRA, A.H.; PEETZ, M.S. Comercialização de antúrios brasileiros nos mercados interno e externo. Antúrios. Fortaleza: Embrapa Agroindústria Tropical, 2005d.

JUNQUEIRA, A.H.; PEETZ, M.S. Mercado interno para os produtos da floricultura brasileira: características, tendências e importância socioeconômica recente. Revista Brasileira de Horticultura Ornamental, Campinas, v.14, n.1, p.37-52, 2008.

LANDGRAF，P.R.C.; PAIVA，P.D.O. Produção de flores cortadas no estado de Minas Gerais. Ciência e Agrotecnologia, v.33, p.120-126, 2009a.

LANDGRAF, P.R.C.; PAIVA, P.D.O. Produção de mudas para jardim no estado de Minas Gerais. Ciência e Agrotecnologia, v.33, p.127-131, 2009b.

LANDGRAF, P.R.C; PAIVA, P. D. O. Agronegócio da floricultura brasileira. Magistra, v. 21, p. 253-261, 2009.

LANDGRAF, P.R.C; PAIVA, P. D. O. Floricultura-Produção e comercialização no estado de Minas Gerais. 1. ed. Lavras: UFLA, 2008. v. 1. 101p

MATOS, C.A.S.; ALVES, F.L. (coord.). NOVO PEDEAG 2007-2025 - Plano Estratégico de Desenvolvimento da Agricultura Capixaba: Estudo Setorial Floricultura. Vitória, ES, dezembro de 2007.

PADULA, A.D.; KÄMPF, A.N.; SLONGO, L.A. (Coord.). Diagnóstico da Cadeia Produtiva de Flores e Plantas Ornamentais do Rio Grande do Sul. SEBRAE/RS e UFRGS. Edição SEBRAE, dezembro de 2003. 160 p.
RIO DE JANEIRO (Governo do Estado). Empresa de Assistência Técnica e Extensão Rural - EMATER. Censo da Floricultura do Estado do Rio de Janeiro 2002/2003. Niterói: EMATER, 2004. 1 CD-Rom.

RIO DE JANEIRO (Governo do Estado). Secretaria de Agricultura e Pecuária (SEAPA). Empresa de Assistência Técnica e Extensão Rural (EMATER). Censo da floricultura de Itaboraí. Rio de Janeiro: SEAPA, 2011.

RIO DE JANEIRO (Governo do Estado). Secretaria de Agricultura e Pecuária (SEAPA). Programa Florescer. Número de produtores, área plantada e valor da produção da floricultura no Estado do Rio de Janeiro. Rio de Janeiro: SEAPA, janeiro de 2014a.

RIO DE JANEIRO (Governo do Estado). Secretaria de Agricultura e Pecuária (SEAPA). Programa Florescer. Produto Interno Bruto (PIB) da floricultura no Estado do Rio de Janeiro, 2010-2013. Rio de Janeiro: SEAPA, janeiro de 2014b.

SECRETARIA DE AGRICULTURA E ABASTECIMENTO DO ESTADO DO PARANÁ (SEAB). Departamento de Economia Rural (DERAL). Valor da Produção da Agropecuária Paranaense, 2008 a 2013. Curitiba/PR, 2014.

SERVIÇO DE APOIO ÀS MICRO E PEQUENAS EMPRESAS NO ESTADO DE ALAGOAS-SEBRAE/AL. Floricultura. Disponível em: $<$ http://www.al.sebrae.com.br/ programas-projetos/default.asp > . Acesso em 27 out.2004.

SERVIÇO DEAPOIO ÀS MICRO E PEQUENAS EMPRESAS NO ESTADO DE PERNAMBUCO - SEBRAE / PE. Diagnóstico da floricultura pernambucana. In Floricultura em Pernambuco, Recife (Edição SEBRAE, Série Agronegócio), 84p., 2002.

SERVIÇO DE APOIO ÀS MICRO E PEQUENAS EMPRESAS NO ESTADO DO RIO DE JANEIRO- SEBRAE / RJ. Diagnóstico da Cadeia Produtiva de Flores e Plantas do Estado do Rio de Janeiro. Rio de Janeiro: SEBRAE / RJ, maio de 2003. Disponível em: $<\underline{\text { http://201.2.114.147/bds/ }}$ BDS.nsf/8104AA054E31F117832572290065B9F4/\$File/ NT000B5F32.pdf $>$ Acesso em 7 jun.2007.

SERVIÇO DE APOIO ÀS MICRO E PEQUENAS EMPRESAS NO ESTADO DO RIO DE JANEIRO- SEBRAE / RJ SEBRAE / RJ. Diagnóstico da Cadeia Produtiva de Flores e Plantas Ornamentais no Estado do Rio de Janeiro. Rio de Janeiro: SEBRAE / RJ, 2003. 